\title{
A Targeted Biopsy during Menstruation for the Definitive Diagnosis of Rectovaginal Endometriosis: A Report of Two Cases
}

\author{
Kosuke Tanaka ${ }^{1}$, Akiko Sasaki ${ }^{1}$, Hideto Egashira ${ }^{1}$, Shinichi Teshima ${ }^{2}$, Karen Kimura ${ }^{1}$, \\ Takashi Nishino ${ }^{1}$, Tomohiko Tazawa ${ }^{1}$, Junichi Tasaki ${ }^{1}$, Chikamasa Ichita ${ }^{1}$, Sakue Masuda ${ }^{1}$, \\ Kazuya Koizumi ${ }^{1}$ and Makoto Kako ${ }^{1}$
}

\begin{abstract}
:
We herein report the definitive diagnosis of rectovaginal endometriosis in two cases. Case 1 involved a 46year-old woman with abdominal pain and hematochezia. The diagnosis after the first and second examinations using lower gastrointestinal (GI) endoscopy was unclear. Differential diagnoses included mucosaassociated lymphoid tissue and colorectal cancer. The third lower GI endoscopy with a targeted biopsy, performed during menstruation, confirmed rectovaginal endometriosis. Case 2 involved a 38-year-old woman with hematochezia. Lower GI endoscopy during menstruation revealed rectovaginal endometriosis. When rectovaginal or bowel endometriosis is suspected, lower GI endoscopy and a targeted biopsy during menstruation can prevent unnecessary surgery.
\end{abstract}

Key words: targeted biopsy, endometriosis, lower gastrointestinal endoscopy, menstruation, rectovaginal endometriosis

(Intern Med 60: 999-1004, 2021)

(DOI: 10.2169/internalmedicine.5430-20)

\section{Introduction}

Rectovaginal endometriosis is a deep-infiltrating endometriosis (DIE) characterized by an endometriotic lesion situated more than $5 \mathrm{~mm}$ below the peritoneum (1-3). In suspected cases, an examination using lower gastrointestinal (GI) endoscopy is necessary to rule out malignancies; however, its accuracy is only about $9 \%$ (4).

We herein report two cases of rectovaginal endometriosis wherein a definitive diagnosis was established by adjusting the timing of lower GI endoscopy and a biopsy to correspond with menstruation.

\section{Case Reports}

This report was approved by the Shonan Kamakura Gen- eral Hospital Institutional Review Board and followed the tenets of the Declaration of Helsinki. Written informed consent was obtained from both patients prior to the publication of this case report and its accompanying images.

\section{Case 1}

A 46-year-old Japanese woman who received treatment at our hospital for endometriosis and uterine myomas presented to the gynecology unit with a chief complaint of abdominal pain. No abnormalities were detected on a physical examination, and the findings of transvaginal sonography were unremarkable. Thus, she was prescribed an analgesic and observed. After one week, the pain subsided, but she developed hematochezia and was admitted.

Lower GI endoscopy revealed a submucosal tumor-like elevation and a stricture extending from the rectum to the sigmoid colon, through which the scope could pass with

${ }^{1}$ Gastroenterology Medicine Center, Shonan Kamakura General Hospital, Japan and ${ }^{2}$ Diagnostic Pathology, Shonan Kamakura General Hospital, Japan

Received: May 26, 2020; Accepted: August 27, 2020; Advance Publication by J-STAGE: October 28, 2020

Correspondence to Dr. Kosuke Tanaka, kosuke.tsssik@gmail.com 


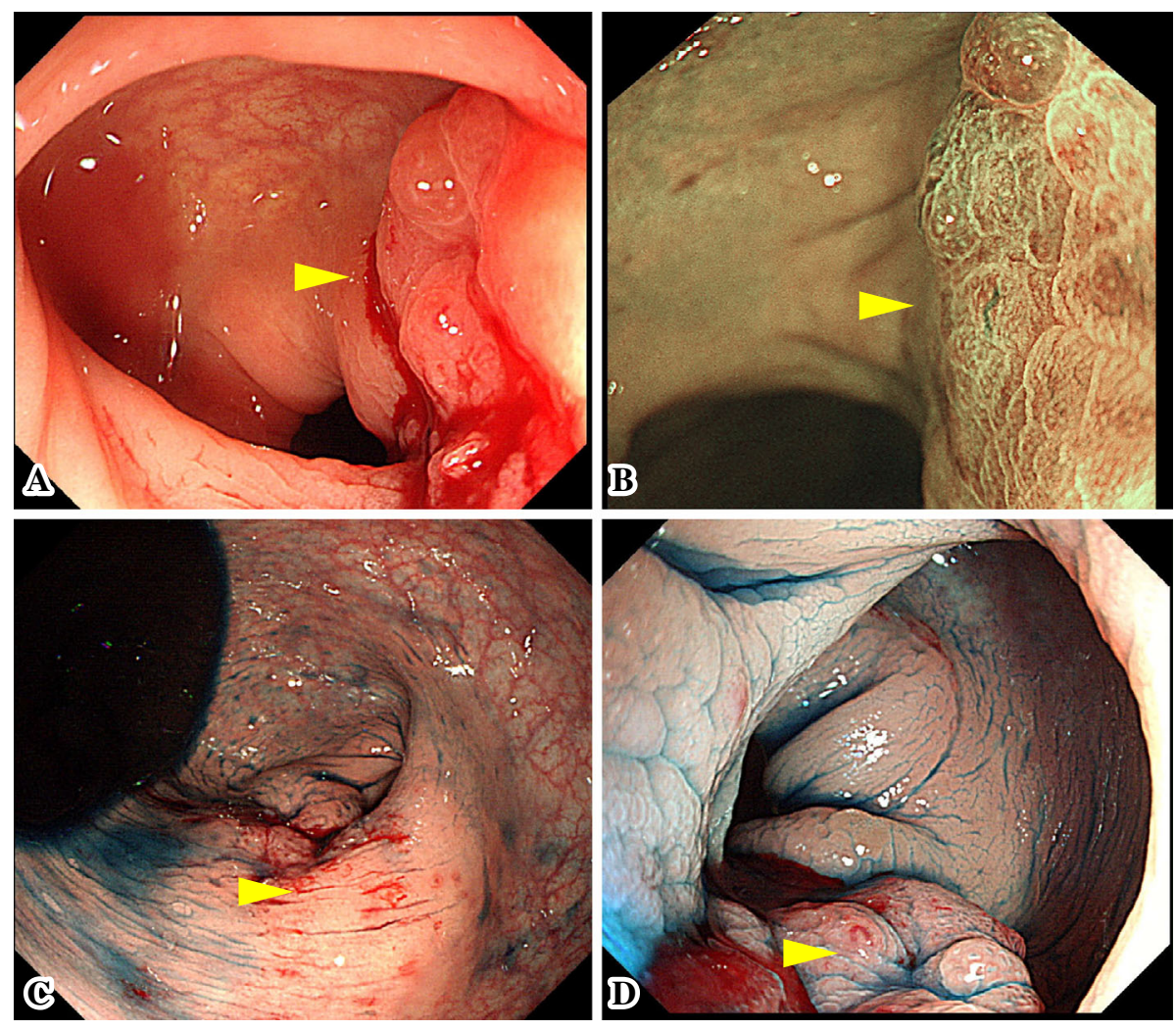

Figure 1. Case 1: Findings of the first and second lower GI endoscopy. (A, B) Initial lower GI endoscopy revealed stenosis from the rectum to the sigmoid colon, although the scope passed with resistance. The lesion was suggested to be type 4 colorectal cancer involving three-fourths of the wall (arrowheads). (C, D) The second lower GI endoscopy showed the same findings (arrowheads). GI: gastrointestinal

some resistance. Dilated short vessels were found on the surface of the lesion, and both the vessels and the surface pattern showed no irregularity. The macroscopic findings suggested type 4 colorectal cancer involving three-fourths of the wall (Fig. 1A, B). Furthermore, granular changes with a cobblestone pattern were observed in the area of stenosis. The first biopsy of the granular nodules was performed without adjusting for the timing of the menstrual cycle, and a pathological diagnosis of lymphoid cell infiltration, which might be mucosa-associated lymphoid tissue, was made.

Three specimens were taken, and none had features suggestive of endometriosis. The tumor marker levels were as follows: carcinoembryonic antigen, $2.8 \mathrm{ng} / \mathrm{mL}$; cancer antigen (CA) 19-9, absent; and CA125, $94.2 \mathrm{U} / \mathrm{mL}$. A second lower GI endoscopy and biopsy were performed during the patient's intermenstrual period and produced the same results (Fig. 1C, D). However, another pathological diagnosis of chronic colitis without malignancy was made based on the second biopsy. Six specimens were acquired from the granular nodules, and none indicated endometriosis. The patient was informed of the possible relationship between hematochezia and the menstrual cycle and consented to undergo endoscopy during menstruation.

During the third endoscopy, we found reddened nodules and a spontaneously bleeding hemorrhagic mucous membrane that had not been observed during the previous endo- scopies. The surface of the nodules appeared redder than they were during the intermenstrual period, with an evident reticulated network of blood vessels. Short, expanded vessels and small, webbed vessels were marked. Scattered nodules were seen on the surface, and there was an enlarged glandular duct with a type 1 pit pattern at its edge, and microcapillaries with small nonstructural depressions were observed at the top of the nodules (Fig. 2A, B). The third biopsy was taken from the reddish granular nodules using Radial Jaw ${ }^{\mathrm{TM}} 4$ Jumbo Cold Polypectomy Forceps (Boston Scientific, Marlborough, USA), which enabled the acquisition of samples from deeper layers.

The pathological findings indicated rectovaginal endometriosis. Endometrial-like glands and stroma, commonly found in the endometrium during the proliferative phase, were seen in the rectal mucosa (Fig. 2C). Immunostaining revealed estrogen receptor positivity, confirming the diagnosis of rectovaginal endometriosis (Fig. 2D). In addition, 6 of the 10 collected specimens had features indicative of endometriosis. Lower GI fluoroscopy revealed irregular unilateral walls (Fig. 2E), and contrast-enhanced computed tomography showed colorectal wall thickening (Fig. 2F). The patient was prescribed dienogest, and her symptoms improved. 


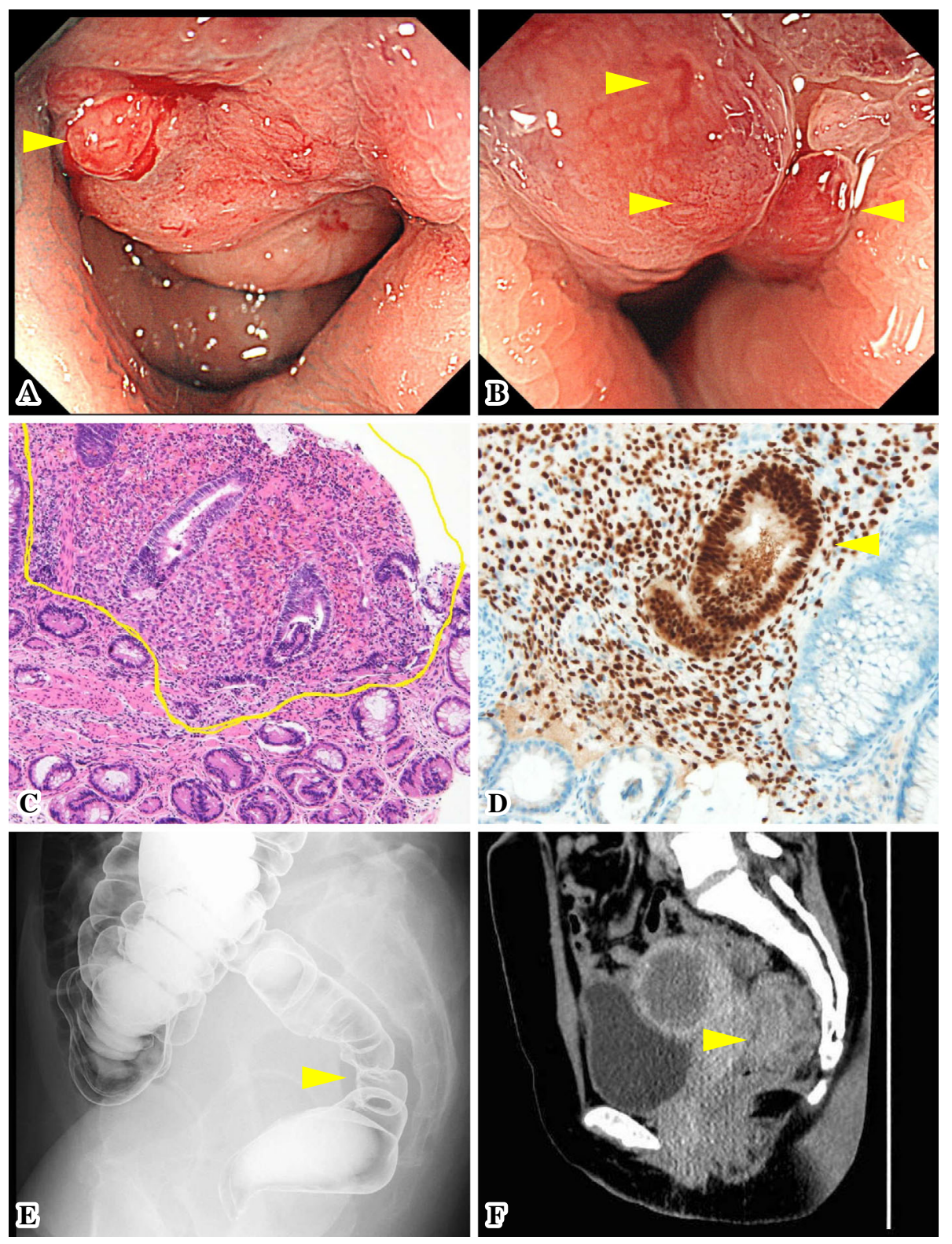

Figure 2. Case 1: Findings of the third lower GI endoscopy, lower GI fluoroscopy, contrast-enhanced CT, and pathological examination. (A, B) The third lower GI endoscopy revealed reddened nodules and a hemorrhagic mucous membrane with spontaneous bleeding. Each expanded short and webbed small blood vessel is marked (arrowheads). (C) A pathological examination revealed rectal mucosa resembling endometrial glands and stroma of the proliferative phase (100 HPF) (inside yellow line). (D) Immunostaining yielded positive findings for estrogen receptor (400 HPF) (arrowhead). (E) Lower GI fluoroscopy revealed an irregular unilateral wall (arrowhead). (F) Contrast-enhanced CT revealed colorectal wall thickening with a contrast effect (arrowhead). CT: computed tomography, GI: gastrointestinal

\section{Case 2}

A 38-year-old Japanese woman with a history of hematochezia during menstruation visited our outpatient unit. Ten months prior to her visit, she underwent lower GI endoscopy in the absence of menstruation; the macroscopic and pathological findings were suggestive of ischemic enteritis. Her tumor marker levels were not checked at that time. Her menstrual cycle lasted approximately four weeks, and she had hematochezia within the first few days. We suspected rectovaginal endometriosis and performed a second lower GI endoscopy during the first day of menstruation. We recognized a protuberance resembling a submucosal tumor and reddened nodules in the rectum (Fig. 3A, B).

The surface of the nodules was deep red, similar to that observed in Case 1, and it had eroded regions and bled spontaneously. A targeted biopsy of the most-reddened nodules was performed. The pathological findings were suggestive of rectovaginal endometriosis. Endometrial-like glands and stroma, usually seen during the menstrual phase in the 

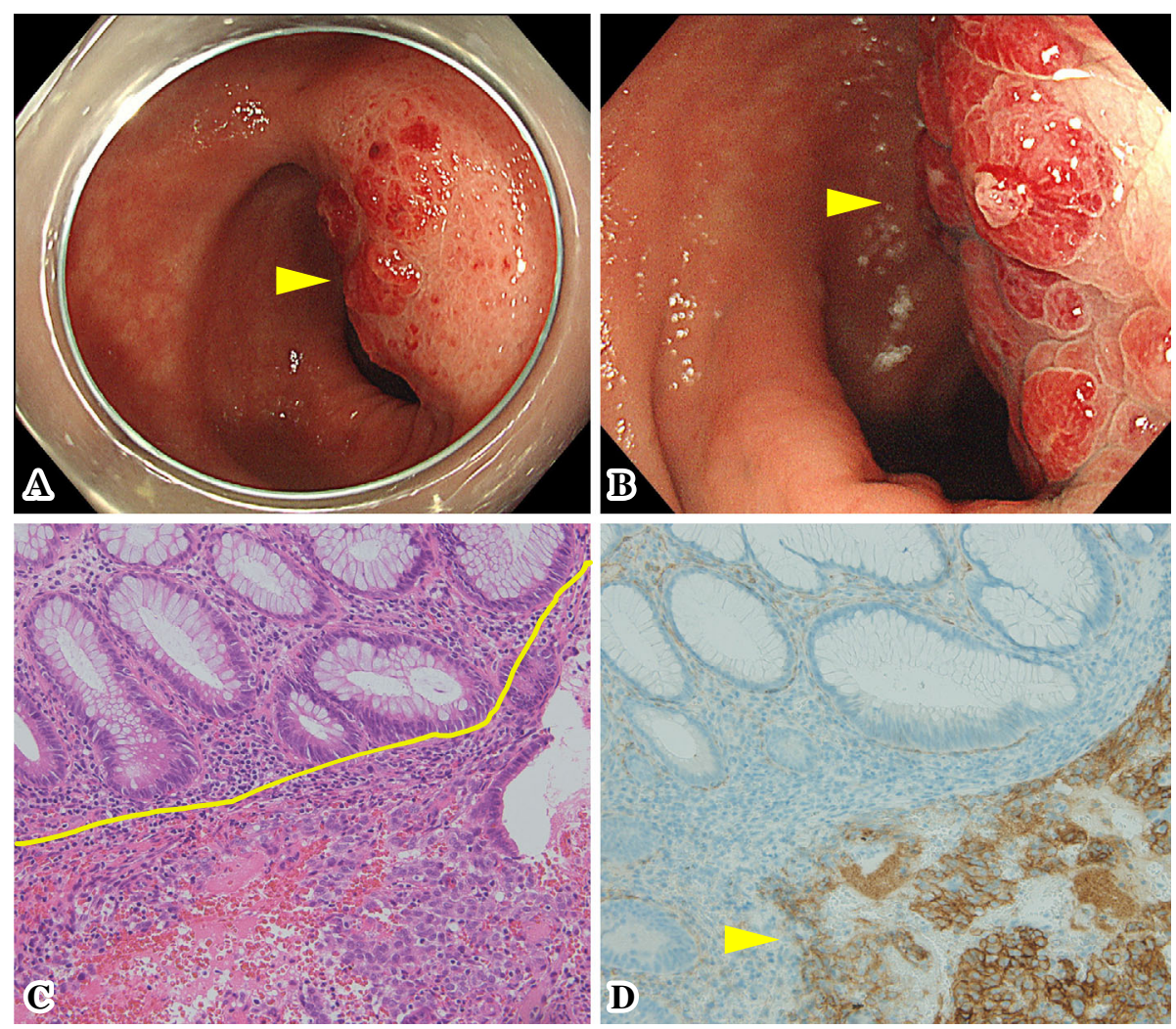

Figure 3. Case 2: Findings of the second lower GI endoscopies. (A, B) Lower GI endoscopy revealed a protuberance that resembled a submucosal tumor and reddened nodule in the rectum (arrowheads). (C) A pathological examination revealed rectal mucosa resembling endometrial glands and stroma seen in the menstrual phase (100 HPF) (under yellow line). (D) Immunostaining yielded positive results for CD10 (400 HPF; arrowhead). GI: gastrointestinal

endometrium, were seen in the rectal mucosa (Fig. 3C). Immunostaining showed CD10 positivity, and a definitive diagnosis of rectovaginal endometriosis was made (Fig. 3D). Findings from all three specimens were suggestive of endometriosis. Accordingly, the diagnosis was established; however, the patient was not placed on medication because she wished to conceive. She became pregnant one month later and did not report any hematochezia until the sixmonth follow-up; she then moved to her parent's house for her delivery.

\section{Discussion}

We herein report two cases of rectovaginal endometriosis diagnosed using lower GI endoscopy and a biopsy performed during menstruation. Rectovaginal endometriosis often presents with submucosal tumor-like elevations, which may not necessarily expose the endometrial tissue to the mucosal surface. However, a diagnostic biopsy may prevent unnecessary surgery, and further innovations in endoscopic biopsy-based diagnoses are desirable.

According to the most common theory of the pathogenesis of endometriosis, the theory of ectopic endometrial cells (Sampson's theory of retrograde menstruation), endometrial cells flow backward through the uterine tubes into the peritoneal cavity during menses (5). In a case series of over 100 women who underwent surgical treatment for endometriosis, the distributions of both peritoneal or deep bowel lesion sites were as follows: rectum, 13-53\%; sigmoid colon, 18$47 \%$; ileum or other small bowels, 2-5\%; and appendix, 3$18 \%$ (6-9). These data show that rectovaginal endometriosis is quite common and is not an unusual finding in our patients. Women with rectovaginal or bowel endometriosis commonly present with GI symptoms, such as diarrhea, constipation, abdominal bloating, and pain (10). These symptoms typically present during menstruation, and they are reportedly seen in only approximately $50 \%$ of cases of endometriosis (10). Other symptoms include rectal bleeding, dyspareunia, and severe dysmenorrhea (11). Treatment options for endometriosis can be medical or surgical, with the former being the first line of treatment. Several factors, such as severity of symptom, patient preferences, adverse effects, treatment efficacy, desire to conceive, and cost, among others, need to be considered when planning treatment.

While there is an increased likelihood of endometriosis in women with pelvic pain, analgesics and hormonal medications are often used for treatment in the absence of a definitive laparoscopic diagnosis (12). For patients who do not wish to conceive, medical treatment should be the first option. Surgery is suggested when patients with pelvic pain are unresponsive to more conservative treatments. A review determined that a woman with severe pain, defined by a visual 
analog scale score of $\geq 7$, benefited from surgery, and the authors described a surgical treatment algorithm based on the depth and size of the rectal disease (13). Therefore, if symptoms persist with substantial endoscopic findings during a longer follow-up period, the surgical treatment algorithm should be followed. Surgery is also chosen when the pathological findings are unclear. It has been reported that some patients may relapse or develop cancer during the course of treatment; thus, surgical resection should be considered in cases of prolonged medical treatment (14). In a previous case series of 78 women who underwent surgery, the rate of accurate diagnoses by a lower GI biopsy was only $9 \%$ (4). In addition, CA125 measurement can be useful in the diagnosis of DIE, with a reported sensitivity and specificity of $36 \%$ and $87 \%$, respectively (15). In the previous study, the rate of the preoperative diagnosis of rectovaginal endometriosis using imaging was $42 \%$, and the rate of a misdiagnosis as an intestinal tumor was $29 \%$ (4). Some studies have suggested that the lower GI tract is rarely assessed to diagnose bowel endometriosis, as lesions that penetrate the GI mucosa are unusual $(10,16,17)$. Lower GI endoscopy is often performed to exclude malignancy or check for bowel stenosis.

This disease is classified as endometrioma type or diffuse endometriosis (18). In the endometrioma type, the endometrial glands at the nodular part of the submucosal mass cause bloody stool during menstruation. Endometrial tissue is often exposed on the mucosal surface and is associated with granular changes that resemble a cobblestone pattern (18). In the diffuse endometriosis type, the ectopic endometrial glands repeatedly bleed into the wall of the intestinal tract, leading to fibrosis and stricture of the intestinal wall due to a lack of extensibility (18). These lesions rarely extend to the mucous membrane, often resulting in stenosis without specific mucosal findings (18). For this reason, a diagnosis using a biopsy may be more difficult in the diffuse endometriosis type than in the endometrioma type (18).

In the cases reported here, case 1 presented with both the endometrioma and diffuse endometriosis types, while case 2 only had the endometrioma type. In cases like case 2 , a biopsy must be performed on the reddened nodules where the uterine mucosa appears to be exposed.

In a recent case series of 17 women with colorectal endometriosis, the rate of an accurate diagnosis by a lower GI biopsy was $47 \%$ (8/17 patients) (19). Colonoscopy showed eccentric wall thickening in $82 \%$ of patients (14/17), and the rate of a definitive diagnosis was higher for patients with surface nodules $(67 \%, 8 / 12$ patients) than for those without nodules (0\%, 0/5 patients) (19). Our cases had similar features, indicating that eccentric wall thickening and nodularity are important for diagnosing rectovaginal endometriosis, although they are not sufficient for a definitive diagnosis. In the abovementioned study (19), the number of endoscopic biopsy specimens was not associated with the diagnostic yield. The median number of biopsy specimens for our cases was 4.5 (range, 3-9), and the diagnostic yield was not proportional to the number of specimens. Furthermore, the previous report did not indicate the timing of the biopsy (e. g. during menstruation).

We recommend that a targeted biopsy be conducted during menstruation because during the non-menstrual period, the surface of the lesion is covered by a dense and smooth layer of intact endometrium. Therefore, even if the endometrium is exposed at the intestinal mucosa, it may be difficult to macroscopically distinguish it from the surrounding tissue. In addition, it may be covered by normal intestinal mucosa because of its lack of ischemic changes. During menstruation, the stratum functionale becomes necrotic and bleeds; this is recognized endoscopically as erythematous granules. During menstruation, ischemic changes cause the epithelium to shed, resulting in a larger exposed area. Thus, the lesions and specimens may show features of endometriosis more clearly, making them easier to recognize. This potentially increases the targeted biopsy diagnosis rate.

For case 1, endoscopic findings were evaluated during the menstrual cycle as well as the intermenstrual period. During the menstrual cycle, reddish nodules with a hemorrhagic mucous membrane, which bled spontaneously, and blood vessels resembling spiral arteries, which were not seen during the intermenstrual period, appeared. The nodules appeared redder and were scattered on the surface. It has been reported that before menstruation, the mucous membrane reddens and appears edematous, and irregularities in the blood vessels disappear (20). During menstruation, fresh blood is found in the mucous membrane (20).

A targeted biopsy may be more effective than conventional one because it facilitates a focused evaluation of the nodules. Even if there are no typical findings on the mucosal surface, submucosal endometrial tissue may be harvested from deeper layers using larger forceps to obtain a larger specimen; this is particularly useful when rectovaginal endometriosis is suspected.

Differential diagnoses include submucosal tumors, such as a GI stromal tumor, malignant lymphoma, disseminated metastatic colorectal cancer, diffuse infiltrating colorectal carcinoma, mesenteric panniculitis, malignant tumor, pelvic inflammatory disease, and inflammatory bowel disease (4). A previous report showed that lipoma is the most common colorectal submucosal tumor, with rectovaginal endometriosis being the fourth-most common (21). In our cases, it was difficult to differentiate between rectovaginal endometriosis, diffuse infiltrating colorectal carcinoma, and malignant lymphoma. Well-timed lower GI endoscopy and a targeted biopsy during menstruation resulted in a definitive diagnosis.

In conclusion, lower GI endoscopy and a biopsy during menstruation aided in making a definitive diagnosis of rectovaginal endometriosis in our patients. Thus, when rectovaginal or bowel endometriosis is suspected, lower GI endoscopy and a targeted biopsy during menstruation can avoid unnecessary surgery.

The authors state that they have no Conflict of Interest (COI). 


\section{References}

1. De Cicco C, Corona R, Schonman R, Mailova K, Ussia A, Koninckx PR. Bowel resection for deep endometriosis: a systematic review. BJOG 118: 285-291, 2011.

2. Koninckx PR, Meuleman C, Demeyere S, Lesaffre E, Cornillie FJ. Suggestive evidence that pelvic endometriosis is a progressive disease, whereas deeply infiltrating endometriosis is associated with pelvic pain. Fertil Steril 55: 759-765, 1991.

3. Chapron C, Bourret A, Chopin N, et al. Surgery for bladder endometriosis: Long-term results and concomitant management of associated posterior deep lesions. Hum Reprod 25: 884-889, 2010.

4. Matukura N, Matsuo Y, Tsuruta O, et al. Two cases of intestinal endometriosis and literature review of the 78 cases in Japan. Gastrointest Endosc 31: 1577-1584, 1989.

5. Sampson JA. Peritoneal endometriosis due to the menstrual dissemination of endometrial tissue into the peritoneal cavity. Am J Obstet Gynecol 14: 422, 1927.

6. Weed JC, Ray JE. Endometriosis of the bowel. Obstet Gynecol 69: 727-730, 1987.

7. Redwine DB. Ovarian endometriosis: a marker for more extensive pelvic and intestinal disease. Fertil Steril 72: 310-315, 1999.

8. Bailey HR, Ott MT, Hartendorp P. Aggressive surgical management for advanced colorectal endometriosis. Dis Colon Rectum 37: 747-753, 1994.

9. Pereira RM, Zanatta A, Preti CD, de Paula FJ, da Motta EL, Serafini PC. Should the gynecologist perform laparoscopic bowel resection to treat endometriosis? Results over 7 years in 168 patients. J Minim Invasive Gynecol 16: 472-479, 2009.

10. Remorgida V, Ferrero S, Fulcheri E, Ragni N, Martin DC. Bowel endometriosis: presentation, diagnosis, and treatment. Obstet Gynecol Surv 62: 461-470, 2007.

11. Chapron C, Santulli P, de Ziegler D, et al. Ovarian endometrioma: Severe pelvic pain is associated with deeply infiltrating endometriosis. Hum Reprod 27: 702-711, 2012.
12. Dunselman GA, Vermeulen N, Becker C, et al. ESHRE guideline: management of women with endometriosis. Hum Reprod 29: 400412, 2014.

13. Abrão MS, Petraglia F, Falcone T, Keckstein J, Osuga Y, Chapron C. Deep endometriosis infiltrating the recto-sigmoid: critical factors to consider before management. Hum Reprod Update 21: 329-339, 2015.

14. Heaps JM, Nieberg RK, Berek JS. Malignant neoplasms arising in endometriosis. Obstet Gynecol 75: 1023-1028, 1990.

15. Koninckx PR, Muyldermans M, Meuleman C, Cornillie FJ. CA 125 in the management of endometriosis. Eur J Obstet Gynecol Reprod Biol 49: 109-113, 1993.

16. Roseau G. Recto-sigmoid endoscopic-ultrasonography in the staging of deep infiltrating endometriosis. World J Gastrointest Endosc 6: $525-533,2014$.

17. Kaufman LC, Smyrk TC, Levy MJ, Enders FT, Oxentenko AS. Symptomatic intestinal endometriosis requiring surgical resection: Clinical presentation and preoperative diagnosis. Am J Gastroenterol 106: 1325-1332, 2011.

18. Izumi Y, Matsunaga H, Kajiwara M, Akashi Y, Miyoshi A. Report of two cases of colo-rectal endometriosis. Jpn J Gastroenterol Surg 27: 932-936, 1994 (in Japanese, Abstract in English).

19. Kyung-Jo Kim, Sang-Su Jung, Suk-kyun Yang, et al. Colonoscopic findings and histologic diagnostic yield of colorectal endometriosis. J Clin Gastroenterol 45: 536-541, 2011.

20. Nagumo H, Adachi S, Egawa $Y$, et al. A case of intestinal endometriosis diagnosed by colonoscopic biopsy. Prog Dig Endosc 84: 174-175, 2014.

21. Sanomura M, Nishida M, Ishihara Y, et al. Diagnosis of submucosal tumor-like protrusion of the colorectum. Stomach and Intestine 55: 701-717, 2020.

The Internal Medicine is an Open Access journal distributed under the Creative Commons Attribution-NonCommercial-NoDerivatives 4.0 International License. To view the details of this license, please visit (https://creativecommons.org/licenses/ by-nc-nd/4.0/).

(C) 2021 The Japanese Society of Internal Medicine Intern Med 60: 999-1004, 2021 